\title{
Crecimiento del cangrejo Callinectes danae (Decapoda: Portunidae) de la Isla de Margarita, Venezuela
}

\author{
Jesylén Castillo ${ }^{1,2}$, Nora Eslava ${ }^{2}$ \& Leo Walter González ${ }^{2}$ \\ 1. Escuela de Ciencias Aplicadas del Mar. Universidad de Oriente, Núcleo Nueva Esparta, Venezuela; \\ jesylencastillo31@gmail.com \\ 2. Área de Biología y Recursos Pesqueros, Instituto de Investigaciones Científicas, Universidad de Oriente, Núcleo \\ Nueva Esparta, Venezuela; neslava20@yahoo.es,1wgc25@gmail.com
}

\author{
Recibido 03-IX-2010. C Corregido 09-III-2011. Aceptado 08-IV-2011.
}

\begin{abstract}
Dana Swimming crab growth Callinectes danae (Decapoda: Portunidae) from Margarita Island, Venezuela. Callinectes danae is a common species captured with crab traps in nearby areas of coastal lagoons in Margarita Island. Although its considerable economic importance as a fishery resource, few studies have been done on population dynamics and its fishery potential in local coastal environments to support decision making in fishery administration. We present growth pattern details of Callinectes danae to better estimate its population size and exploitation feasibility. For this, we analyzed a total of 3623 specimens that were monthly captured in crab pots by artisanal fishermen in Las Marites lagoon, from October 2007 to September 2008. The length-weight ratio was determined, and growth parameters estimated from both length and weight curves of the von Bertalanffy model. The general sex ratio showed no significant difference between males and females $\left(\chi^{2}=0.04, p>0.05\right)$. However, values of slopes $b$ between males and females were significantly different $\left(\mathrm{t}_{\mathrm{s}}=2.75, \mathrm{p}<0.05\right)$, as well as intercepts $a\left(\mathrm{t}_{\mathrm{s}}=2.44, \mathrm{p}<0.05\right)$. Thus, the length-weight ratio was determined separately: $\mathrm{W}=7.48 \mathrm{e}^{-5 *} \mathrm{~L}^{2.98}$ for males and $\mathrm{W}=1.21 \mathrm{e}^{-4 *} \mathrm{~L}^{2.87}$ for females, indicating a negative allometric growth in both sexes. Growth parameters were established as: $\mathrm{L}_{\infty}=134.80 \mathrm{~mm}, \mathrm{~W}_{\infty}=166.04 \mathrm{~g}$ and $\mathrm{k}=0.86 / \mathrm{yr}$ for males; $\mathrm{L}_{\infty}=122.35 \mathrm{~mm}, \mathrm{~W}_{\infty}=118.45 \mathrm{~g}$ and $\mathrm{k}=0.63 / \mathrm{yr}$ for females. Lifespan was estimated at 3.05 years for males and 4.24 years for females. We concluded that Callinectes danae is a species with short lifespan and moderately rapid growth. The coefficient of variation values $(\mathrm{CV})$, of the phi-prime growth performance index $\left(\varnothing^{\prime}\right)$, showed a different growth pattern compared to those obtained in other regions. We propose that a management strategy will be the periodical review of the minimum capture size for fishing area, after the great variability found in growth parameters. Rev. Biol. Trop. 59 (4): 1525-1535. Epub 2011 December 01.
\end{abstract}

Key words: growth, dana swimcrab, Callinectes danae, Portunidae, Margarita Island, Venezuela.

En la dinámica de poblaciones sometidas a explotación, el conocimiento y caracterización del crecimiento individual es de suma importancia pues este proceso, junto con la supervivencia, determina la magnitud de la disponibilidad del recurso. En este sentido, es evidente que al obtener un conocimiento detallado sobre el patrón de crecimiento de una especie se lograrán estimaciones más precisas sobre el tamaño de la población y su disponibilidad para la explotación, o por lo menos con márgenes de variabilidad conocidos (Arreguin et al. 1991).
Se puede definir el crecimiento de manera cuantitativa de varias formas, entre ellos se encuentra el modelo de von Bertalanffy. Aunque la fisiología de los crustáceos es muy diferente a la de los peces, el crecimiento, en la mayoría, se adecua al modelo de crecimiento de von Bertalanffy (Musick \& Frazer 1999).

Las especies del género Callinectes Stimpson, 1860 representan un recurso pesquero comercial importante en aguas del Atlántico y del Pacífico, alcanzando elevados valores en los mercados de consumo (Villasmil \& 
Mendoza 2001). Estados Unidos de América es uno de los principales consumidores e importadores de jaiba que se captura a lo largo de la costa este y sur del Atlántico. En México, la jaiba es un recurso pesquero artesanal que se explota con mayor intensidad en el litoral del Pacífico (Rosas \& Navarrete 2008). En Venezuela, es explotado principalmente en el Lago de Maracaibo. Esta pesquería artesanal se inició en el año 1969 y representa una de las más importantes del país, tanto por el valor comercial de las capturas como, por el número de empleos generados en la fase de extracción y procesamiento (Villasmil 1994). Según las estadísticas del Instituto Socialista de Pesca y Acuicultura (INSOPESCA), el desembarque en Venezuela durante 2007 fue de 17 725t. En la Isla de Margarita la pesca está restringida en zonas cercanas a lagunas costeras, y a la oferta y demanda por parte de intermediarios quienes la comercializan en restaurantes y supermercados de manera limitada (González 2008, com. pers.).

Callinectes danae Smith, 1869 constituye, indudablemente, un recurso de considerable importancia económica entre los crustáceos comestibles; sin embargo, pocos estudios han sido publicados sobre la biología, ecología y potencialidad pesquera en ambientes costeros de Venezuela que amerita información de índole periódica de los parámetros de crecimiento indispensables en la evaluación y toma de decisiones en la administración pesquera. Por tal razón, se decidió realizar esta investigación con el fin de contribuir al conocimiento de la dinámica poblacional de este recurso para su mejor aprovechamiento de manera sustentable de la flota artesanal de La Isleta, la cual está ubicada en el municipio García, en la costa sur de la Isla de Margarita, su costa presenta alternados acantilados y playas que registran una intensa actividad pesquera, y el mar circundante está caracterizado por un sistema de corrientes que mantiene en constante renovación la masa de agua (Lárez 2004).

\section{MATERIALES Y MÉTODOS}

Se realizaron muestreos mensuales desde octubre 2007 hasta septiembre 2008 y se analizó un total de 3623 ejemplares de $C$. danae en La Isleta, durante la actividad de pesca de la flota artesanal (96 embarcaciones) que opera en áreas cercanas a la Laguna de Las Marites (1053'25” N - 635 5' 33 ” W). Del total, 10 embarcaciones de $7 \mathrm{~m}$ de eslora con motor fuera de borda realizaron faenas tres veces al día, desde las 7am hasta las $12 \mathrm{pm}$, utilizando cada una 45 nasas cangrejeras rectangulares (40$45 \mathrm{~cm}$ de largo, $30-35 \mathrm{~cm}$ de ancho y $30-40 \mathrm{~cm}$ de altura), unidas a través de una cuerda (enyugue de nasas) a una distancia de 10 brazadas entre nasas.

A cada uno de los especímenes se les midió el ancho del caparazón (L), definido como la distancia comprendida entre las dos espinas agudas laterales, para lo que se dispuso de una tabla graduada con apreciación de $1 \mathrm{~mm}$. Así mismo se estableció el peso total (P) mediante una balanza digital, con capacidad de $3 \mathrm{~kg}$ y apreciación de $1 \mathrm{~g}$. Para la identificación del sexo se siguió el criterio descrito por Hendrickx (1995) según el cual, el abdomen de los machos es estrecho en forma de "T" invertida y en las hembras es en forma de "U" invertida.

Para analizar diferencias significativas respecto a la proporción sexual $\delta:$ ㅇ se aplicó la prueba Chi-cuadrado (Zar 1996) $(\alpha=0.05$, $\left.\chi^{2}=3.841\right)$ con corrección de Yates para continuidad (Steel \& Torrie 1985).

Relación longitud-peso: Se determinó la relación talla-peso mediante la ecuación propuesta por Ricker (1975): $\mathrm{P}=\mathrm{a}^{*} \mathrm{~L}^{\mathrm{b}}$; donde $\mathrm{P}$ es el peso total en $\mathrm{g}$, L es el ancho del caparazón en mm, $a$ es la intersección con el eje de las ordenadas y $b$ es la pendiente de la curva. Las constantes $a$ y $b$ fueron estimadas por el método de los mínimos cuadrados, previa linearización de los datos mediante una transformación logarítmica (Ricker 1973). Se determinó la 
existencia de diferencias significativas entre los coeficientes de machos y hembras ( $a$ y $b$ ), y se probó la hipótesis del crecimiento isométrico (Ricker 1975) mediante una prueba t student (Walpole \& Myers 1989) con un nivel de significación de $\alpha=0.05$.

Parámetros de crecimiento: La estimación del crecimiento se basó en el análisis de la distribución de frecuencia de longitudes (anchos del caparazón). Para ello se uso el software FiSAT (Gayanilo et al. 1996). En primer lugar, se estimó una longitud asintótica $\left(\mathrm{L}_{\infty}\right)$ aplicando el método de Powell (1979) y Wetherall (1986), que permitió determinar el coeficiente de crecimiento $(\mathrm{k})$ a través de la rutina ELEFAN I. En segundo lugar, se calculó el $t_{\mathrm{o}}$ de acuerdo a la fórmula anotada por Pauly (1979): $\log _{10}\left(-\mathrm{t}_{\mathrm{o}}\right)=-0.3922-0.2752 * \log _{10} \mathrm{~L}_{\infty}{ }^{-}$ $1.038 * \log _{10} \mathrm{k}$; donde $\mathrm{t}_{\mathrm{o}}$ es la edad teórica en que el organismo tiene talla cero, $\mathrm{L}_{\infty}$ es el ancho del caparazón asintótico o ancho promedio máximo y $\mathrm{k}$ es el coeficiente de crecimiento o coeficiente de curvatura. Para el cálculo de la longevidad se utilizó la ecuación de Taylor (1958): $\mathrm{A}_{0.95}=\mathrm{t}_{\mathrm{o}}+2.996 / \mathrm{k}$; donde $\mathrm{A}_{0.95}$ es la edad límite o tiempo requerido para alcanzar el 95\% del ancho del caparazón máximo promedio $\left(\mathrm{L}_{\infty}\right)$. El peso asintótico se estimó mediante la fórmula anotada por Csirke (1980): $\mathrm{P}_{\infty}=\mathrm{a}^{*} \mathrm{~L}_{\infty}{ }^{\mathrm{b}}$; donde $\mathrm{P}_{\infty}$ es el peso asintótico o peso máximo promedio.

Una vez ajustados $\mathrm{L}_{\infty}, \mathrm{P}_{\infty}, \mathrm{k} \mathrm{y}_{\mathrm{o}}$, tanto en machos como en hembras, se determinaron las curvas de crecimiento en longitud y en peso adecuadas al modelo de von Bertalanffy (1938), según las siguientes ecuaciones: $\mathrm{L}_{\mathrm{t}}=\mathrm{L}_{\infty}\left[1-\mathrm{e}^{-\mathrm{k}(\mathrm{t}-\mathrm{t} \mathrm{t})}\right] ; \mathrm{P}_{\mathrm{t}}=\mathrm{P}_{\infty}\left[1-\mathrm{e}^{-\mathrm{k}(\mathrm{t}-\mathrm{t} \mathrm{t})}\right]^{\mathrm{b}}$. Las curvas de crecimiento, de longitud (ancho del caparazón) para ambos sexos, fueron comparadas a través de un análisis de varianza de doble vía con un nivel de significación de 0.05 (Sokal \& Rohlf 1969). Los parámetros de crecimiento estimados de $C$. danae, fueron comparados con resultados obtenidos por otros investigadores en diferentes regiones, mediante el índice phi prima (Ø') de Munro \& Pauly (1983): $\varnothing^{\prime}=\log _{10} k+2 \log _{10} L_{\infty}$ y el criterio de Sparre
\& Venema (1995) quienes establecen que el coeficiente de variación (CV) no debe exceder del $4 \%$ para poder asumir que los resultados son estadísticamente similares.

\section{RESULTADOS}

Los machos mostraron longitudes (anchos del caparazón) entre 48 y 130mm (media $97.20 \pm 11.25 \mathrm{~mm}$ ) y pesos que oscilaron entre 7 y $135 \mathrm{~g}$ (media $64.85 \pm 20.69 \mathrm{~g}$ ). Por otra parte, las hembras presentaron longitudes de 54 a $121 \mathrm{~mm}$ (media $82.96 \pm 7.35 \mathrm{~mm}$ ) y pesos que oscilaron entre 11 y $109 \mathrm{~g}$ (media $39.36 \pm 9.85 \mathrm{~g}$ ) (Cuadro 1). La proporción sexual de machos y hembras se desvió de la igualdad a lo largo de todo el período de muestreo; sin embargo, para la totalidad del período de estudio la proporción sexual global fue 1:1 $\quad\left(\chi^{2}=0.04\right.$, $\mathrm{p}>0$.05) (Cuadro 2).

Relación longitud-peso: Los valores de la pendiente $b$ entre machos y hembras presentaron diferencias significativas (ts $=2.75, \mathrm{p}<0.05$ ) igualmente las intersecciones $a$ (ts $=2.44$, $\mathrm{p}<0.05$ ); razón por la cual la relación longitudpeso se estableció por separado, quedando en los machos: $\mathrm{P}=7.48 \mathrm{e}^{-5 *} \mathrm{~L}^{2.98} \mathrm{y}$ en las hembras: $\mathrm{P}=1.21 \mathrm{e}^{-4 *} \mathrm{~L}^{2.87}$.

Parámetros de crecimiento: La longitud asintótica estimada fue $\mathrm{L}_{\infty}=134.80 \mathrm{~mm}$ para machos y $\mathrm{L}_{\infty}=122.35 \mathrm{~mm}$ para hembras (Fig. 1); el coeficiente de crecimiento fue $\mathrm{k}=0.86 / \mathrm{año}$ en machos y k=0.63/año en hembras; la edad teórica $\mathrm{t}_{\mathrm{o}}=-0.43$ años en machos y $\mathrm{t}_{\mathrm{o}}=-0.51$ años en hembras. La longevidad o edad límite $\left(\mathrm{A}_{0.95}\right)$ calculada fue de 3.05 años para machos y 4.24 años para hembras.

La comparación de las curvas de crecimiento en longitud (ancho del caparazón) y peso por sexo, a partir del análisis de varianza, demostró que hay diferencia estadísticamente significativa entre machos y hembras tanto en las curvas de longitud $\left(\mathrm{F}_{\mathrm{S}}=1128.36, \mathrm{p}<0.05\right)$, como en las de peso $\left(\mathrm{F}_{\mathrm{S}}=115.06, \mathrm{p}<0.05\right)$; lo que conllevó a establecer las curvas de crecimiento por sexo separado ajustadas al modelo 


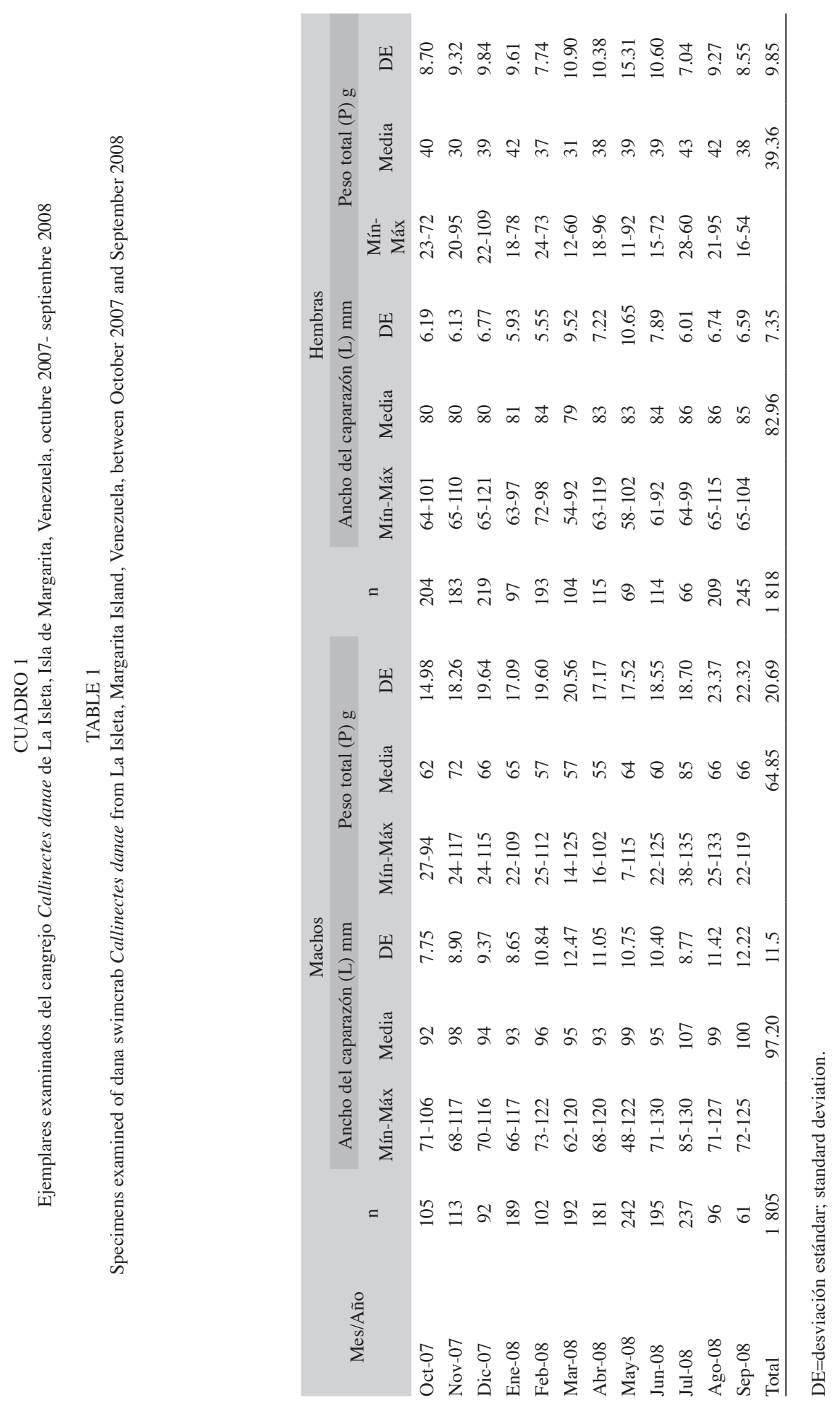


CUADRO 2

Proporción sexual mensual del cangrejo Callinectes danae, octubre 2007-septiembre 2008

TABLE 2

Monthly sex ratio of dana swimcrab Callinectes danae between October 2007 and September 2008

\begin{tabular}{|c|c|c|c|c|c|c|}
\hline Mes & Año & $\hat{0}$ & q & Total & $\widehat{\delta}: P$ & $\chi^{2}$ \\
\hline Octubre & 2007 & 105 & 204 & 309 & $0.5: 1.0$ & $31.08 *$ \\
\hline Noviembre & 2007 & 113 & 183 & 296 & $0.6: 1.0$ & $16.08^{*}$ \\
\hline Diciembre & 2007 & 92 & 219 & 311 & $0.4: 1.0$ & $51.05^{*}$ \\
\hline Enero & 2008 & 189 & 97 & 286 & $1.9: 1.0$ & $28.95^{*}$ \\
\hline Febrero & 2008 & 102 & 193 & 295 & $0.5: 1.0$ & $27.46^{*}$ \\
\hline Marzo & 2008 & 192 & 104 & 296 & $1.8: 1.0$ & $25.57 *$ \\
\hline Abril & 2008 & 181 & 115 & 296 & $1.6: 1.0$ & $14.27 *$ \\
\hline Mayo & 2008 & 242 & 69 & 311 & $3.5: 1.0$ & $95.13 *$ \\
\hline Junio & 2008 & 195 & 114 & 309 & $1.7: 1.0$ & $20.71 *$ \\
\hline Julio & 2008 & 237 & 66 & 303 & $3.6: 1.0$ & $9538 *$ \\
\hline Agosto & 2008 & 96 & 209 & 305 & $0.5: 1.0$ & $41.13 *$ \\
\hline Septiembre & 2008 & 61 & 245 & 306 & $0.2: 1.0$ & $109.44 *$ \\
\hline Total & & 1805 & 1818 & 3623 & $1.0: 1.0$ & 0.04 \\
\hline
\end{tabular}

*Proporción sexual significativamente diferente de 1:1 $(\mathrm{p}<0.05) .\left(\chi^{2}\right.$ tabulado=3.841).

$*$ Sex ratio significantly different $1: 1(\mathrm{p}<0.05) .\left(\chi^{2}\right.$ tabulate $\left.=3.841\right)$

$$
\begin{aligned}
& Y=28.87(-0.214) * X \\
& L_{\infty}=134.80 \\
& r=-0.99
\end{aligned}
$$
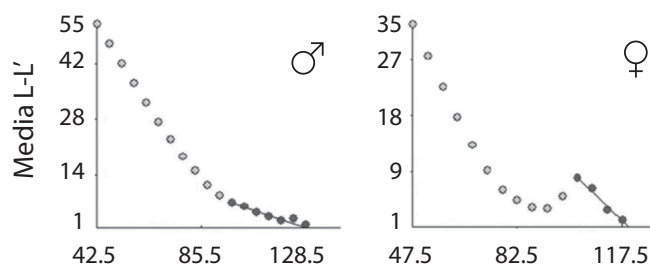

Longitud inferior ( $\mathrm{L}^{\prime} \mathrm{mm}$ )

Fig. 1. Estimación de $\mathrm{L}_{\infty}$ aplicando el modelo de Powell (1979) y Wetherall (1986) del cangrejo Callinectes danae ( $\lesssim \mathrm{y}$ ㅇ).

Fig. 1. Estimation of $\mathrm{L}_{\infty}$ applying the Powell (1979) and Wetherall (1986) model of dana swimcrab Callinectes danae ( $\supset$ and $\circ$ ).

de von Bertalanffy. Las curvas de crecimiento en longitud de machos y hembras describieron curvas de tipo exponencial; se observó un rápido crecimiento en el primer año de vida, para luego disminuir a medida que alcanzaba la longitud asintótica (Fig. 2).

El peso asintótico $\left(\mathrm{P}_{\infty}\right)$ fue obtenido usando las constantes $a$ y $b$ de la relación talla-peso y las longitudes asintóticas $\left(\mathrm{L}_{\infty}\right)$ estimadas. Los valores obtenidos fueron: $\mathrm{P}_{\infty}=166.04 \mathrm{~g}$ en machos y $\mathrm{P}_{\infty}=118.45 \mathrm{~g}$ en hembras, los cuales fueron reemplazados en la ecuación de crecimiento en peso. Las curvas de crecimiento en peso evidenciaron una curva tipo sigmoidea, donde se observó un crecimiento lento en el primer año de vida, luego se aceleró y posteriormente se produjo una inflexión hasta acercarse a su peso máximo (Fig. 3).

El coeficiente de variación obtenido tanto en machos $(\mathrm{CV}=9 \%)$ como en hembras $(\mathrm{CV}=12 \%)$, permitió establecer que hay diferencia en el patrón de crecimiento de la especie C. danae (Cuadro 3).

\section{DISCUSIÓN}

Las tallas más grandes encontradas $(\hat{\jmath}=130 \mathrm{~mm}, q=121 \mathrm{~mm})$ fueron similares a 


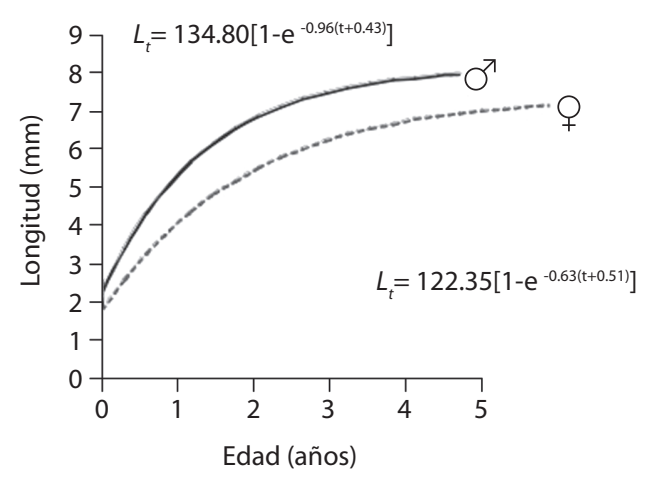

Fig. 2. Curvas de crecimiento en longitud del cangrejo Callinectes danae ( $\hat{\sigma} \mathrm{y}$ †) ajustadas al modelo de von Bertalanffy (1938).

Fig. 2. Growth curves in length of dana swimcrab Callinectes danae ( $\delta$ and + ) according to the von Bertalanffy model (1938).

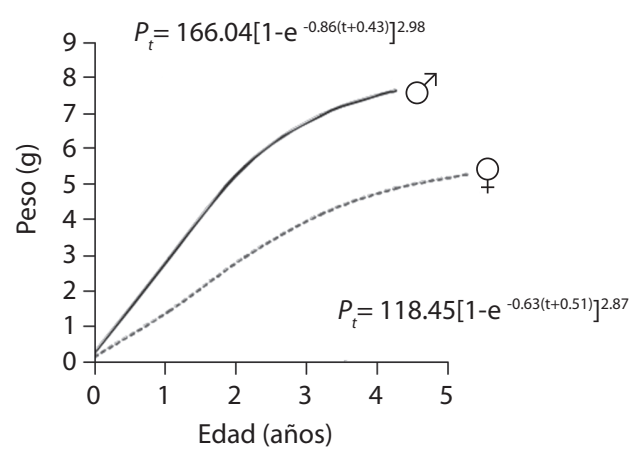

Fig. 3. Curvas de crecimiento en peso del cangrejo Callinectes danae ( $\hat{\sigma}$ y + ) $)$ según el modelo de von Bertalanffy (1938)

Fig. 3. Growth curves in weight of dana swimcrab Callinectes danae ( $\delta$ and + ) according to the von Bertalanffy model (1938).

CUADRO 3

Parámetros de crecimiento del cangrejo Callinectes danae estimados por diferentes autores

TABLE 3

Growth parameters estimated of dana swimcrab Callinectes danae from different authors

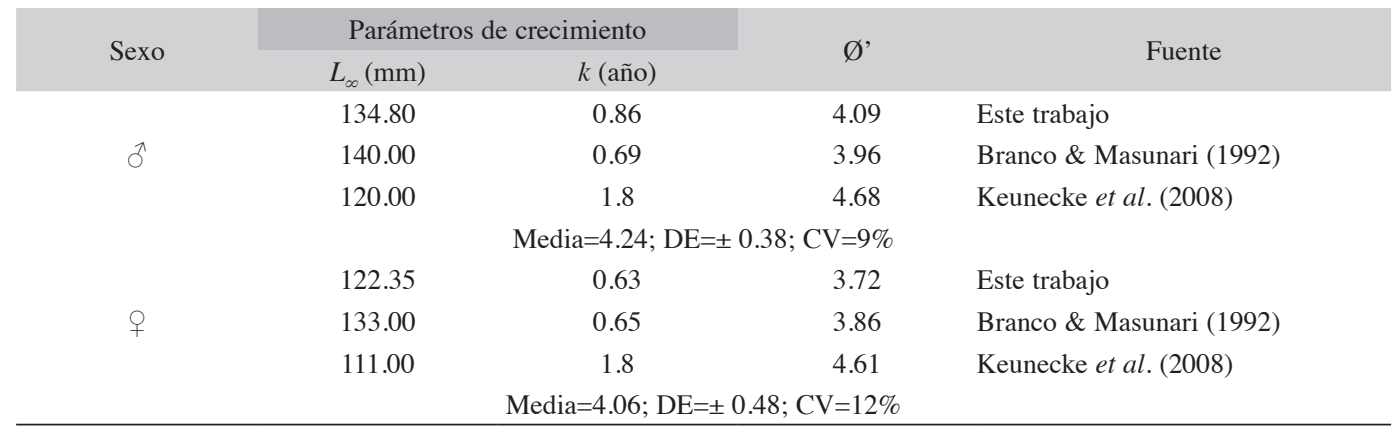

$\mathrm{DE}=$ desviación estándar; standard deviation.

$\mathrm{CV}=$ coeficiente de variación; coefficient of variation.

las registradas por Branco \& Masunari (1992) en la Laguna de Concepción, Florianópolis, Brasil $(\hat{o}=135 \mathrm{~mm}, \quad+=115 \mathrm{~mm})$; sin embargo, se encuentran por encima de lo registrado por Baptista et al. (2005) $(\hat{\delta}=93.2 \mathrm{~mm}$, $q=86 \mathrm{~mm}$ ) en el balneario Shangri-lá, Paraná, Brasil, y Keunecke et al. (2008) $(\widehat{\partial}=120 \mathrm{~mm}$, $q=113 \mathrm{~mm}$ ) en la Bahía de Guanabara, Río de Janeiro, Brasil. Los pesos mínimos y máximos, en ambos sexos de $C$. danae, difieren de lo encontrado por Keunecke et al. (2008), el cual reportó un intervalo de pesos (ठ̊:de 2 a $109 \mathrm{~g}$, q:de 2 a $81 \mathrm{~g}$ ), que se encontra por debajo de los valores obtenidos en el presente estudio, esta diferencia podría ser debida a que los especímenes fueron obtenidos con red de arrastre tipo camaronera, donde la muestra estuvo representada principalmente por jóvenes; mientras que 
en el presente estudio los individuos fueron capturados con nasas cangrejeras colocadas en zonas cercanas a la Laguna Las Marites, razón por las que se obtuvo un alto porcentaje de individuos adultos. Por otro lado, la selección efectuada por el tamaño de malla de las nasas, se pudo considerar como un caso de selección que tiene lugar permitiendo o impidiendo el paso a través de una abertura, que controla la retención y la fuga de los cangrejos de acuerdo a su tamaño (Pope et al. 1983).

La proporción sexual no se desvió significativamente de 1:1, posiblemente, por estar relacionada con el proceso reproductivo continuo. Durante el muestreo se pudo observar que la cópula se presentó durante todo el año en el área de pesca cercana a la Laguna de Las Marites, lo que hace suponer que la Laguna es la zona de crecimiento y reproducción. Resultado similar fue obtenido en $C$. danae por Branco \& Masunari (2000). Por otro lado, Van Engel (1958) comprobó que la cópula de $C$. sapidus se realiza en aguas de baja salinidad, y luego las hembras fecundadas migran hacia aguas de mayor salinidad; mientras que los machos permanecen dentro de su ambiente local, copulando con otras hembras.

Relación longitud-peso: El valor de $b$, para machos $(b=2.98)$ y hembras $(b=2.87)$, indicó un crecimiento alométrico negativo, lo cual significa que, a medida que los individuos crecen se modifican sus proporciones corporales, siendo, en este caso, menos pesados para la longitud que se tiene. Dicho resultado coincide con lo reportado por Pereira-Barros \& Travassos (1975) $(b=2.65)$ y Branco \& Thives (1991) ( $b=2.89)$ para hembras de $C$. danae. Estudios de crecimiento en hembras de C. bellicosus (Molina-Ocampo 2001) y C. bocourti (Costa et al. 1980), mostraron un crecimiento alométrico negativo. Molina-Ocampo (2001), anotó que este tipo de crecimiento es debido a las bajas tasas de alimentación y mayor gasto energético en la temporada reproductiva. Un crecimiento isométrico $(b=3)$ indica que, a medida que crecen mantienen sus proporciones corporales, como señala Baptista et al. (2005) para la misma especie. De la misma manera, Nevárez et al. (2003) y Hernández \& Arreola (2007), observaron crecimiento isométrico en $C$. arcuatus y $C$. bellicosus; expresando, estos últimos investigadores, que los machos del género Callinectes, eventualmente pueden tener un sesgo hacia el crecimiento alométrico, debido a la presencia de hembras en la temporada reproductiva.

La relación longitud-peso mostró que los machos alcanzan mayores tallas y pesos que las hembras. Esto en los braquiuros, parece ser una generalidad asociada con el proceso reproductivo debido a que la hembras detienen su crecimiento para destinar gran parte de la energía a la reproducción, mientras que los machos continúan creciendo aún después de alcanzar la madurez sexual; y la cópula se realiza cuando la hembra está mudada por lo que el macho que la toma debe tener mayor tamaño para protegerla de depredadores; mientras que se regenera el exoesqueleto (Sastry 1983). El mayor peso de los machos, también, puede ser explicado por la diferencia en el tamaño de los apéndices quelados, los cuales son más robustos en los machos. Pereira-Barros \& Travassos (1975) y Costa et al. (1980), estudiando el crecimiento de $C$. danae y $C$. bocourti, indican que ocurre dimorfismo sexual, en cuanto al peso, los machos son más pesados que las hembras. Este factor conllevaría a considerar que los resultados obtenidos en la presente investigación corroboran las características biológicas de la especie.

Parámetros de crecimiento: Las longitudes asintóticas $\left(\mathrm{L}_{\infty}\right)$ estimadas de $C$. danae en este estudio fueron diferentes a las encontradas por Williams (1974) en la costa este de los Estados Unidos ( $\delta=140 \mathrm{~mm}$; $+=120 \mathrm{~mm})$, Branco \& Masunari (1992) en Florianópolis, Brasil $(\hat{\delta}=140 \mathrm{~mm}$; $\uparrow=133 \mathrm{~mm})$ y Keunecke et al. (2008) en Río de Janeiro, Brasil $(\hat{\delta}=120 \mathrm{~mm}, q=111 \mathrm{~mm})$. Igualmente, difieren de las presentadas por Rosas \& Navarrete (2008) para Callinectes sapidus de la Bahía de Chetumal, México $(\widehat{\jmath}-q=231.50 \mathrm{~mm})$ y Ferreira \& D'Incao (2008) para la misma especie en 
la Laguna Los Patos, Brasil $(\hat{\delta}=162.71 \mathrm{~mm}$; $q=157.78 \mathrm{~mm}$ ). En tal sentido, se puede considerar que las diferencias son debidas, posiblemente, a las zonas geográficas, a la aplicación de métodos distintos en la determinación de edad y crecimiento, y a la disparidad en el diseño de muestreo (González 2005). El índice de curvatura (k) estimado para ambos sexos, indica un crecimiento moderadamente rápido, similar a lo señalado por Branco \& Masunari (1992) $(\widehat{\jmath}=0.69 /$ año,,$+=0.65 /$ año $)$, pero difiere con lo reportado por Keunecke et al. (2008) (1.8/año para ambos sexos). Este índice es útil para caracterizar la vulnerabilidad potencial de las poblaciones a una mortalidad excesiva (Musick \& Frazer 1999).

Estos parámetros de crecimiento pudieran tener una respuesta fisiológica explicada por la relación alométrica existente entre el índice metabólico y el peso corporal, dado que las especies pequeñas ingieren alimentos y respiran a mayor velocidad por unidad de peso corporal que las especies más grandes (Hill et al. 2006). Según Sparre \& Venema (1995), los parámetros de crecimiento difieren de una especie a otra, y pueden variar de una población a otra dentro de una misma especie, es decir los parámetros de crecimiento de una especie en particular pueden tener valores diferentes durante su ciclo de vida. Asimismo, mencionan que cohortes sucesivos pueden crecer de forma diferente según las condiciones ambientales y, además, los parámetros de crecimiento varían a menudo en función del sexo.

La diferencia de la edad límite en machos y hembras, puede ser atribuible al mayor valor de $\mathrm{k}$ de los machos quienes alcanzan la longitud asintótica en menor tiempo que las hembras; lo que indica que el crecimiento de las hembras es más lento y por consiguiente tienen un ciclo de vida más largo para lograr alcanzar la longitud asintótica. Al mismo tiempo, el hecho de que las hembras presenten mayor longevidad podría estar relacionado con el comportamiento de los machos. Silva et al. (2004) estudiando el braquiuro Uca rapax indicó que los machos pasan más tiempo expuestos en las áreas de alimentación, compitiendo con otros machos por el territorio y cortejando a las hembras, este comportamiento los hace más susceptibles a la adversidad y a los depredadores. Esta diferencia sexual en la edad de estos organismos, también tendría relación con la calidad del hábitat, la disponibilidad de alimento o estrés fisiológico (Bond \& Backup 1983).

La curva de crecimiento en longitud del tipo exponencial suele ser muy rápido al principio, cuando el organismo es muy joven, pero se va haciendo más lento a medida que aumenta la edad, y a medida que éste alcanza el tamaño o la longitud máxima promedio que cada individuo puede alcanzar. No obstante, el crecimiento en peso sigue un patrón diferente, ya que describe una curva del tipo sigmoideo; debido, probablemente, a que en las etapas tempranas de vida parte de la energía es destinada a la maduración de las gónadas (Csirke 1980).

El alto valor del coeficiente de variación del índice de phi prima ( $\left.\varnothing^{\prime}\right)$ por sexo, indica diferencia en los patrones de crecimiento de Callinectes danae, esto podría deberse a características de hábitat, estimaciones a partir de distintos métodos de análisis de datos, tamaño de la muestra, modalidad de captura y selectividad de los artes de pesca (Rosas \& Navarrete 2008). Sin embargo, Pauly \& Munro (1984) anotaron que dentro de una familia, los valores de ( $\left.\varnothing^{\prime}\right)$ están normalmente distribuidos y, que en las especie de una misma familia sus valores son similares.

\section{AGRADECIMIENTOS}

Expresamos nuestro más sincero agradecimiento a los pescadores de la comunidad de La Isleta, Orangel Antón, Juancho, Moncho y Alexis por su apoyo logístico en la obtención de las muestras biológicas. A Francisco Guevara por su apoyo en el laboratorio de Biología Pesquera del IIC-UDONE. A Clark Casler por las aclaratorias sobre el recurso.

\section{RESUMEN}

Las especies del género Callinectes sostienen pesquerías importantes en varias partes del mundo. En el 
occidente de Venezuela, Callinectes sapidus es capturado con palangre y sostiene una pesquería artesanal importante en el Lago de Maracaibo; mientras que en la Isla de Margarita, Callinectes danae es capturado con nasa en zonas cercanas a lagunas costeras y su pesca está limitada por la oferta y la demanda. Estos recursos son de considerable importancia económica entre los crustáceos comestibles; sin embargo, pocos estudios han sido reportados sobre la dinámica poblacional y potencialidad pesquera en ambientes costeros de Venezuela que contribuyan a la toma de decisiones en la administración pesquera. En este documento se presenta información de los parámetros de crecimiento del cangrejo Callinectes danae Smith, 1869, con el fin de obtener un conocimiento detallado del patrón de crecimiento y lograr estimaciones más precisas sobre el tamaño de la población y su disponibilidad para la explotación. Se analizó una muestra constituida por 3623 ejemplares capturados con nasa cangrejera por la flota artesanal, entre octubre 2007 y septiembre 2008. Se estableció la relación longitud-peso y se estimaron los parámetros de crecimiento de las curvas en longitud y peso del modelo de von Bertalanffy. La proporción sexual global mostró que no existe diferencia significativa entre machos y hembras $\left(\chi^{2}=0.04\right.$, $\mathrm{p}>0.05$ ). Los valores de las pendientes $b$ entre machos y hembras presentaron diferencias significativas $\left(\mathrm{t}_{\mathrm{s}}=2.75\right.$, $\mathrm{p}<0.05)$ igualmente los interceptos a $\left(\mathrm{t}_{\mathrm{s}}=2.44, \mathrm{p}<0.05\right)$; por lo que se estableció la relación longitud-peso por separado: $\mathrm{P}=7.48 \mathrm{e}^{-5 *} \mathrm{~L}^{2.98}$ para machos y $\mathrm{P}=1.21 \mathrm{e}^{-4 *} \mathrm{~L}^{2.87}$ para hembras, evidenciándose un crecimiento alométrico negativo en ambos sexos. Los parámetros de crecimiento quedaron establecidos en: $\mathrm{L}_{\infty}=134.80 \mathrm{~mm}, \mathrm{P}_{\infty}=166.04 \mathrm{~g}$ y k=0.86/año para machos; $\mathrm{L}_{\infty}=122.35 \mathrm{~mm}, \mathrm{P}_{\infty}=118.45 \mathrm{~g}$ y $\mathrm{k}=0.63 / \mathrm{año}$ para hembras. La longevidad fue de 3.05 años para machos y 4.24 años para hembras. Se concluye que Callinectes danae es una especie de vida corta con crecimiento moderadamente rápido, y los valores del coeficiente de variación (CV) del índice de crecimiento phi prima ( $\left.\varnothing^{\prime}\right)$ demostraron diferencia de los patrones de crecimiento obtenidos en otras regiones. Como medida de manejo se recomienda considerar la talla mínima de captura por zona de pesca y revisar periódicamente las normativas, debido a la gran variabilidad en el crecimiento.

Palabras clave: crecimiento, cangrejo, Callinectes danae, Portunidae, Isla Margarita, Venezuela.

\section{REFERENCIAS}

Arreguín, F., J.A. Sánchez \& O. Defeo. 1991. Análisis del crecimiento de la almeja amarilla (Mesodesma mactroides) de la costa uruguaya en base a datos de composición por longitudes. Frente Marítimo 9: 75-81.

Baptista, C., M. Pinheiro, A. Blankesteiyn \& C. Borzone. 2005. Biologia populacional e reproductiva de Callinectes danae Smith (Decapoda: Portunidae) no Balneário Shangrí-lá, Pontal do Paraná, Paraná, Brasil. Rev. Bras. Zool. 22: 446-453.

Von Bertalanffy, L. 1938. A quantitative theory of organic growth. Hum. Biol. 10: 181-213.

Bond, G. \& L. Backup. 1983. O cultivo de Macrobrachium borellii (Nobili, 1896) e de Macrobrachium potiuna (Müller, 1880) em laboratório (Crustacea, Decapoda, Palaemonidae). Rev. Bras. Biol. 43: 177-190.

Branco, J. \& S. Masunari. 1992. Crescimento de Callinectes danae (Decapoda: Portunidae) da lagoa da Conceiçao, Florianápolis, Santa Catarina, Brasil. Rev. Bras. Zool. 9: 53-66.

Branco, J. \& S. Masunari. 2000. Reproductive ecology of the blue crab Callinectes danae Smith 1869, in the Conceição Lagoon system, Santa Catarina Isle, Brazil. Rev. Bras. Biol. 60: 17-27.

Branco, J. \& A. Thives. 1991. Relação peso/ largura, fator de condição e tamanho de primeira maturação de Callinectes danae Smith 1869 (Crustacea: Decapoda) no Manguezal do Itacorubi, SC, Brasil. Arq. Biol. Tecnol. 34: 415-424.

Costa, F.J.C.B., I.V. Nascimento \& M.F.P. Sa. 1980. Estudo biométrico do siri gurjaú, Callinectes bocourti A. Milne Edwards, 1879, da Lagoa Menguaba. Bol. Núcl. Ests. Cis. Mar., Maceió. 2: 5-9.

Csirke, J. 1980. Introducción a la dinámica de poblaciones de peces. FAO, Documento Técnico de Pesca 192. Roma, Italia.

Ferreira, L.S. \& F. D'Incao. 2008. Crescimento de Callinectes sapidus (Crustacea, Decapoda, Portunidae) no estuário da laguna dos Patos, Rio Grande do Sul, Brasil. Iheringia. Ser. Zool. Porto Alegre 98: 70-77.

Gayanilo, F., P. Sparre \& D. Pauly. 1996. Stock assessment tools users manual. FAO, 8. Roma, Italia.

González, L.W. 2005. Análisis de la pesquería artesanal de la sardina Sardinella aurita, Valenciennes, 1847 (Pisces: Clupeidae) de la región nororiental de Venezuela. Bol. Inst. Oceanogr. Venez. 24: 111-128.

Hendrickx, M. 1995. Cangrejos. Guía FAO para la identificación de especies para los fines de pesca. FAO, Roma, Italia.

Hernández, L. \& J. Arreola. 2007. Estructura de tallas y crecimiento de los cangrejos Callinectes arcuatus y C. bellicosus (Decapoda: Portunidae) en la laguna costera Las Guásimas, México. Rev. Biol. Trop. 55: 226-233. 
Hill, R., M. Wyse \& M. Anderson. 2006. Fisiología animal. Panamericana, Caracas.

Keunecke, K., F. D'Incao, F. Moreira, D. Silva \& J. Verani. 2008. Idade e crescimento de Callinectes danae e $C$. ornatus (Crustacea: Decapoda) na Baía de Guanabara, Rio de Janeiro, Brasil. Iheringia. Ser. Zool. Porto Alegre 98: 231-235.

Lárez, D. 2004. Variación de la estructura comunitaria del fitoplancton en el área pesquera Porlamar-La Isleta, Isla de Margarita, Venezuela; en el período febrerojulio 2002. Tesis de Licenciatura, Universidad de Oriente, Boca de Río, Venezuela.

Molina-Ocampo, R. 2001. Jaiba del Estado de Sonora Callinectes bellicosus, p. 329-248. In Sustentabilidad y pesca responsable en México. Evaluación y manejo. INP/SEMARNAP, Sonora, México.

Munro, J. \& D. Pauly. 1983. A simple method for comparing growth of fishes and invertebrates. Fishbyte 1: $5-6$.

Musick, J. \& N. Frazer. 1999. Planificación del manejo para especies longevas. Contribución $\mathrm{N}^{\circ} 2353$ del Virginia Institute of Marine Science: 61-67.

Nevárez, M., J. López, C. Cervantes, E. Miranda, R. Morales \& M. Anguiano. 2003. Evaluación biológica y pesquera de las jaibas Callinectes bellicosus y Callinectes arcuatus (Brachyura: Decapoda: Portunidae) en las bahía de Guásimas y Lobos, Sonora, México, p. 143-151. In M.E. Hendrickx (ed.). Contribuciones al estudio de los crustáceos del Pacífico Este 3. Instituto de Ciencias del Mar y Limnología, UNAM, México.

Pauly, D. 1979. Theory and management of tropical multispecies stocks: a review with emphasis on the Southeast Asian demersal fisheries. ICLARM Studies and Review, Manila, Philippines.

Pauly, D. \& J.L. Munro. 1984. Once more on the comparison of growth in fish and invertebrates. Fishbyte 2: 21 .

Pereira-Barros, J.B. \& I. Travassos. 1975. Informes sobre a pesca e biologia do siri tinga (Callinectes danae) e guajaú (Callinectes bocourti), na Lagoa Mundaú, Maceió, Alagoas. Superintendência do Desenvolvimento do Nordeste, Departamento de Recursos Pesqueiros, Recife, Brasil. 9.
Pope, J., A. Margetts, J. Hamley \& E. Akyüz. 1983. Manual de métodos para la evaluación de las poblaciones de peces. Parte 3. Selectividad del arte de pesca. FAO, Documento Técnico de Pesca 41, Roma, Italia.

Powell, D. 1979. Estimation of mortality and growth parameters for the length frequency of a catch. Report procès-V Réunion Conseil International pour L'Exploitation de la Mer. 175: 167-169.

Ricker, W. 1973. Linear regressions in fishery research. J. Fish. Res. Board. Can. 30: 409-434.

Ricker, W. 1975. Computation and interpretation of biological statistics of fish populations. Bull. Fish. Res. Board. Can. 191: 382.

Rosas, C. \& A. Navarrete. 2008. Parámetros poblacionales de la jaiba azul Callinectes sapidus (Rathbunae, 1896) en la Bahía de Chetumal, Quintan Roo, México. Rev. Biol. Mar. Oceanog. 43: 247-253.

Sastry, A. 1983. Ecological aspects of reproduction, p. 179270. In D.E. Bliss (ed.). The biology of Crustaceae. Academic, Nueva York, EEUU.

Silva, D., D. Silva \& M. Negreiros. 2004. Somatic growth of the mudflat fiddler crab Uca rapax (Smith, 1870) (Brachyura: Ocypodidae) from two subtropical mangroves in Brazil. Universidad y Ciencia 20: 15-22.

Sokal, R. \& F. Rohlf. 1969. Biometry. W.H. Freeman and Co., San Francisco, EEUU.

Sparre, P. \& S. Venema. 1995. Introducción a la evaluación de los recursos pesqueros tropicales. Parte 1. FAO, Documento Técnico de Pesca 306: Revisión 1. Valparaíso, Chile.

Steel, R. \& J. Torrie. 1985. Bioestadística: Principios y procedimientos. Mc. Graw-Hill, Latinoamericana S.A., Bogotá, Colombia.

Taylor, C. 1958. Cod growth and temperature. Journal of Conseil International pour L'Exploitation de la Mer. 23: 366-370.

Van Engel, W. 1958. The blue crab and its fishery in Chesapeake Bay. Reproduction, early development, growth migration. Commer. Fish. Rev. 24: 6-17.

Villasmil, L. 1994. Aspectos taxonómicos y biológicospesqueros del recurso cangrejo del género Callinectes, Stimpson, 1880 (Crustacea: Portunidae) en el 
Lago de Maracaibo, Venezuela. Tesis de Maestría, Universidad de Oriente, Cumaná, Venezuela.

Villasmil, L. \& J. Mendoza. 2001. Las pesquería del cangrejo Callinectes sapidus (Decapoda:Brachyura) en el Lago de Maracaibo, Venezuela. Interciencia 26: 301-306.

Walpole, R. \& R. Myers. 1989. Probabilidad y estadística para ingenieros. Interamericana, México.
Wetherall, J. 1986. A new method for estimating growth and mortality parameters from length-frequency data. Fishbyte 4: 12-14.

Williams, A. 1974. Shrimp lobster and crabs of the Atlantic coast of the Eastern Unites States, Maine to Florida. Smithsonian Institution, Washington D.C., EEUU.

Zar, J. 1996. Biostatistical analysis. Prentice Hall, Nueva Jersey, EEUU. 
